\title{
Le problème infrarouge pour l'électron habillé non relativiste dans un champ magnétique
}

\author{
Laurent Amour, Jérémy Faupin† Benoît Grébertł Jean-Claude Guillot ${ }^{\S}$
}

\section{Résumé}

Nous considérons un électron non relativiste interagissant avec un champ magnétique classique dans la direction $x_{3}$ et un champ électromagnétique quantifié. Le système est invariant par translation suivant $x_{3}$ et l'Hamiltonien correspondant admet une décomposition $H \simeq \int_{\mathbb{R}}^{\oplus} H\left(P_{3}\right) d P_{3}$. Pour une impulsion $P_{3}$ fixée suffisamment petite, nous montrons que $H\left(P_{3}\right)$ possède un état fondamental dans la représentation Fock si et seulement si $E^{\prime}\left(P_{3}\right)=0$, où $P_{3} \mapsto E^{\prime}\left(P_{3}\right)$ est la dérivée de l'application $P_{3} \mapsto E\left(P_{3}\right)=\inf \sigma\left(H\left(P_{3}\right)\right)$. Lorsque $E^{\prime}\left(P_{3}\right) \neq 0$, nous obtenons l'existence d'un état fondamental dans une représentation non équivalente à la représentation Fock. Ce résultat est valable pour des valeurs suffisamment petites de la constante de couplage.

\section{Abstract}

The infrared problem for the dressed non-relativistic electron in a magnetic field.

We consider a non-relativistic electron interacting with a classical magnetic field pointing along the $x_{3}$-axis and with a quantized electromagnetic field. The system is translation invariant in the $x_{3}$-direction and the corresponding Hamiltonian has a decomposition $H \simeq \int_{\mathbb{R}}^{\oplus} H\left(P_{3}\right) d P_{3}$. For a fixed momentum $P_{3}$ sufficiently small, we prove that $H\left(P_{3}\right)$ has a ground state in the Fock representation if and only if $E^{\prime}\left(P_{3}\right)=0$, where $P_{3} \mapsto E^{\prime}\left(P_{3}\right)$ is the derivative of the map $P_{3} \mapsto$ $E\left(P_{3}\right)=\inf \sigma\left(H\left(P_{3}\right)\right)$. If $E^{\prime}\left(P_{3}\right) \neq 0$, we obtain the existence of a ground state in a non-Fock representation. This result holds for sufficiently small values of the coupling constant.

\section{Abridged english version}

We consider a non-relativistic electron of charge $e$ and mass $m$ interacting with a classical magnetic field pointing along the $x_{3}$-axis, an electrostatic potential, and the quantized electromagnetic field in the Coulomb gauge. The position and the momentum of the electron are denoted respectively by $x=\left(x_{1}, x_{2}, x_{3}\right)$ and $p=\left(p_{1}, p_{2}, p_{3}\right)=-i \nabla_{x}$. The classical magnetic field is of the form $\left(0,0, b\left(x^{\prime}\right)\right)$, where $x^{\prime}=\left(x_{1}, x_{2}\right)$ and $b\left(x^{\prime}\right)=\left(\partial a_{2} / \partial x_{1}\right)\left(x^{\prime}\right)-\left(\partial a_{1} / \partial x_{2}\right)\left(x^{\prime}\right)$. Here $a\left(x^{\prime}\right)$ is a vector potential. The electrostatic potential is denoted by $V\left(x^{\prime}\right)$. The quantized electromagnetic field in the Coulomb gauge is defined by (1.2), where $\rho$ is a real ultraviolet cutoff function satisfying (1.3),

\footnotetext{
*Laboratoire de Mathématiques EDPPM, FRE-CNRS 3111, Université de Reims, Moulin de la Housse - BP 1039, 51687 REIMS Cedex 2, France. laurent.amour@univ-reims.fr

${ }^{\dagger}$ Institut for Matematiske Fag, Aarhus Universitet, Ny Munkegade, 8000 Aarhus C, Denmark. faupin@imf.au.dk. Supported by the Centre for Theory in Natural Science

¥Laboratoire de Mathématiques Jean Leray, UMR-CNRS 6629, Université de Nantes, 2, rue de la Houssinière, 44072 Nantes Cedex 3, France. benoit.grebert@univ-nantes.fr

$\S$ Centre de Mathématiques Appliquées, UMR-CNRS 7641, Ecole polytechnique, 99128 Palaiseau Cedex, France. guillot@cmapx.polytechnique.fr
} 
$\epsilon_{1}(k)$ and $\epsilon_{2}(k)$ are polarization vectors orthogonal to each other and to $k$, and $a_{\lambda}^{*}(k)$ and $a_{\lambda}(k)$ are the usual creation and annihilation operators obeying the canonical commutation relations (1.5). The Pauli Hamiltonian $H_{g}$ associated to the system we consider acts on $\mathcal{H}_{\mathrm{el}} \otimes \mathcal{H}_{\mathrm{ph}}$, where $\mathcal{H}_{\mathrm{el}}=\mathrm{L}^{2}\left(\mathbb{R}^{3} \times \mathbb{Z}_{2}\right)$ is the Hilbert space for the electron, and $\mathcal{H}_{\mathrm{ph}}$ is the symmetric Fock space constructed over $\mathrm{L}^{2}\left(\mathbb{R}^{3} \times \mathbb{Z}_{2}\right)$ for the photons. The Hamiltonian $H_{g}$ is then formally given by (1.1), where the charge of the electron $e$ is replaced by a coupling parameter $g$ in the terms containing the quantized electromagnetic field. The Hamiltonian for the photons in the Coulomb gauge is given by (1.4), and $\sigma=\left(\sigma_{1}, \sigma_{2}, \sigma_{3}\right)$ is the 3-component vector of the Pauli matrices. Noting that $H_{g}$ formally commutes with the operator of total momentum in the direction $x_{3}, P_{3}=p_{3}+\mathrm{d} \Gamma\left(k_{3}\right)$, one can show (see [3]) that $H_{g}$ has a decomposition

$$
H_{g}=\int_{\mathbb{R}}^{\oplus} H_{g}\left(P_{3}\right) d P_{3} .
$$

If $P_{3}$ is fixed, $H_{g}\left(P_{3}\right)$ acts on $\mathrm{L}^{2}\left(\mathbb{R}^{2} \times \mathbb{Z}_{2}\right) \otimes \mathcal{H}_{\mathrm{ph}}$ and is formally given by (1.6). The infrared cutoff Hamiltonian $H_{g, \sigma}\left(P_{3}\right)$ is defined by replacing the integral over $\left\{k \in \mathbb{R}^{3}\right\}$ in (1.2) by the integral over $\left\{k \in \mathbb{R}^{3},|k| \geq \sigma\right\}$. We set $E_{g}\left(P_{3}\right)=\inf \sigma\left(H_{g}\left(P_{3}\right)\right)$ and $E_{g, \sigma}\left(P_{3}\right)=\inf \sigma\left(H_{g, \sigma}\left(P_{3}\right)\right)$. The electronic Hamiltonian is $h(b, V)=\sum_{j=1,2} \frac{1}{2 m}\left(p_{j}-e a_{j}\left(x^{\prime}\right)\right)^{2}-\frac{e}{2 m} \sigma_{3} b\left(x^{\prime}\right)+V\left(x^{\prime}\right)$, and we define $e_{0}=\inf \sigma(h(b, V))$ and $e_{1}=\inf \left[\sigma(h(b, V)) \backslash\left\{e_{0}\right\}\right]$. We make the following hypothesis:

$\left(\mathbf{H}_{\mathbf{0}}\right) e_{0}$ is an isolated eigenvalue of multiplicity 1.

Proposition Assume that $\left(\mathbf{H}_{\mathbf{0}}\right)$ holds. Then there exist $g_{0}>0$ and $P_{0}>0$ such that for all $0<|g| \leq g_{0}$, for all $P_{3}, k_{3} \in \mathbb{R}$ such that $\left|P_{3}\right| \leq P_{0},\left|P_{3}+k_{3}\right| \leq P_{0}$, for all $0 \leq \sigma \leq\left(e_{1}-e_{0}\right) / 2$, for all $\delta>0$,

$$
\left|E_{g, \sigma}^{\prime}\left(P_{3}+k_{3}\right)-E_{g, \sigma}^{\prime}\left(P_{3}\right)\right| \leq \mathrm{C}_{\delta}\left|k_{3}\right|^{\frac{1}{4}-\delta},
$$

where $\mathrm{C}_{\delta}$ is a positive constant depending on $\delta$ but independent of $\sigma$.

Remark Our proof follows the scheme of [16, 8].

Let us define a function $f: \mathbb{R}^{3} \times \mathbb{Z}_{2} \rightarrow \mathbb{C}$ by

$$
f(k, \lambda)=\frac{g}{2 m} \frac{\rho(k) \epsilon_{\lambda}^{3}(k)}{k_{3}|k|^{1 / 2}} \frac{E_{g}\left(P_{3}-k_{3}\right)-E_{g}\left(P_{3}\right)}{E_{g}\left(P_{3}-k_{3}\right)-E_{g}\left(P_{3}\right)+|k|} .
$$

We note that if $P_{3} \mapsto E_{g}\left(P_{3}\right)$ is of class $\mathrm{C}^{1+\delta}$ with $\delta>0$, the property $E_{g}\left(P_{3}-k_{3}\right)-E_{g}\left(P_{3}\right) \geq$ $-3|k| / 4$ (see [3]) implies that the function $f$ is in $\mathrm{L}^{2}\left(\mathbb{R}^{3} \times \mathbb{Z}_{2}\right)$ if and only if the derivative $E_{g}^{\prime}\left(P_{3}\right)$ vanishes. As in [4], we consider a renormalized Hamiltonian $H_{g}^{\text {ren }}\left(P_{3}\right)$, defined by the formal expression $H_{g}^{\text {ren }}\left(P_{3}\right)=W(i f) H_{g}\left(P_{3}\right) W(i f)^{*}$, where for $h \in \mathrm{L}^{2}\left(\mathbb{R}^{3} \times \mathbb{Z}_{2}\right), W(h)$ is the Weyl operator, $W(h)=e^{i \Phi(h)}$, with $\Phi(h)=\left(a^{*}(h)+a(h)\right) / \sqrt{2}$. We then obtain the equation (2.3) which defines $H_{g}^{\text {ren }}\left(P_{3}\right)$ no matter whether $f$ is in $\mathrm{L}^{2}\left(\mathbb{R}^{3} \times \mathbb{Z}_{2}\right)$ or not. Note that in $(2.3)$ we have set $A_{j}\left(x^{\prime}, 0\right)=$ $\Phi\left(h_{j}\left(x^{\prime}\right)\right)$. Our main result is

Theorem Assume that $\left(\mathbf{H}_{\mathbf{0}}\right)$ holds. Then there exist $g_{0}>0$ and $P_{0}>0$ such that for all $0<$ $|g| \leq g_{0}$ and $0<\left|P_{3}\right| \leq P_{0}$,

(i) $H_{g}\left(P_{3}\right)$ has a ground state if and only if $E_{g}^{\prime}\left(P_{3}\right)=0$.

(ii) $H_{g}^{\text {ren }}\left(P_{3}\right)$ has a ground state. 


\section{Remark}

1. The previous proposition is used both in the proof of (i) and in the proof of (ii). More precisely, we use $(*)$ with $\sigma=0$ to show the absence of a ground state for $H_{g}\left(P_{3}\right)$ when $E_{g}^{\prime}\left(P_{3}\right) \neq 0$ : arguing as in [10, Lemma 2.6], the key point of our proof is to obtain a contradiction when assuming the existence of a ground state $\Phi_{g}\left(P_{3}\right)$, thanks to the property (2.4). On the other hand, to prove the existence of a ground state for $H_{g}^{\text {ren }}\left(P_{3}\right)$, and for $H_{g}\left(P_{3}\right)$ in the case $E_{g}^{\prime}\left(P_{3}\right)=0$, we follow [3], using $(*)$ with $\sigma>0$ in order to bound the number of photons in the state $\Phi_{g, \sigma}\left(P_{3}\right)$ uniformly in $\sigma$. Here $\Phi_{g, \sigma}\left(P_{3}\right)$ denotes a ground state of the infrared cutoff Hamiltonian $H_{g, \sigma}\left(P_{3}\right)$.

2. Our result extends to the Pauli-Fierz model describing dressed mobile atoms and ions (see $[1,2,14])$, replacing the condition $E_{g}^{\prime}\left(P_{3}\right)=0$ in the theorem by $Q \nabla E_{g}(P)=0$, where $Q$ is the total charge of the atomic system, and $E_{g}(P)$ is the infimum of the spectrum of the reduced Hamiltonian $H_{g}(P)$ at a fixed total momentum $P$.

\section{Définition du modèle et hypothèses}

Nous considérons un électron, traité comme une particule quantique non relativiste, en interaction avec un champ magnétique classique dans la direction $x_{3}$ et le champ électromagnétique quantifié en jauge de Coulomb. L'espace de Hilbert pour l'électron est $\mathcal{H}_{\mathrm{el}}:=\mathrm{L}^{2}\left(\mathbb{R}^{3} \times \mathbb{Z}_{2}\right)$. L'espace de Hilbert pour le champ de photons est l'espace de Fock symétrique construit à partir de $\mathrm{L}^{2}\left(\mathbb{R}^{3} \times \mathbb{Z}_{2}\right)$, c'està-dire $\mathcal{H}_{\mathrm{ph}}:=\mathbb{C} \oplus \bigoplus_{n=1}^{\infty} S_{n}\left[\mathrm{~L}^{2}\left(\mathbb{R}^{3} \times \mathbb{Z}_{2}\right)^{\otimes^{n}}\right]$, où $S_{n}$ désigne la projection orthogonale sur l'espace des fonctions symétriques. Le système que l'on considère est associé à l'opérateur hamiltonien de Pauli $H_{g}$ agissant dans $\mathcal{H}_{\mathrm{el}} \otimes \mathcal{H}_{\mathrm{ph}}$, défini formellement par

$$
H_{g}=\frac{1}{2 m}\left(p-e a\left(x^{\prime}\right)-g A(x)\right)^{2}-\frac{e}{2 m} \sigma_{3} b\left(x^{\prime}\right)-\frac{g}{2 m} \sigma \cdot B(x)+V\left(x^{\prime}\right)+H_{\mathrm{ph}} .
$$

Dans cette définition, les unités sont choisies de telle façon que $\hbar=c=1$, où $\hbar$ est la constante de Planck divisée par $2 \pi$ et $c$ est la vitesse de la lumière. Les paramètres $e$ et $m$ représentent respectivement la charge et la masse de l'électron, et dans les termes contenant le champ électromagnétique quantifié, $e$ est remplacé par une constante de couplage notée $g$. Les opérateurs position et impulsion de l'électron sont notés respectivement $x=\left(x_{1}, x_{2}, x_{3}\right)$ et $p=\left(p_{1}, p_{2}, p_{3}\right)=-i \nabla_{x}$. La variable $x^{\prime}$ est définie par $x^{\prime}=\left(x_{1}, x_{2}\right), \sigma=\left(\sigma_{1}, \sigma_{2}, \sigma_{3}\right)$ désigne le vecteur des matrices de Pauli, et $V\left(x^{\prime}\right)$ est un potentiel électrique. Le champ magnétique classique est de la forme $\left(0,0, b\left(x^{\prime}\right)\right)$, où $b\left(x^{\prime}\right)=\left(\partial a_{2} / \partial x_{1}\right)\left(x_{1}, x_{2}\right)-\left(\partial a_{1} / \partial x_{2}\right)\left(x_{1}, x_{2}\right)$ et $a\left(x^{\prime}\right)$ est un potentiel vecteur. Le champ électromagnétique quantifié en jauge de Coulomb s'écrit

$$
\begin{aligned}
& A(x)=\frac{1}{2 \pi} \sum_{\lambda=1,2} \int \frac{\epsilon_{\lambda}(k)}{|k|^{1 / 2}} \rho(k)\left[e^{-i k \cdot x} a_{\lambda}^{*}(k)+e^{i k \cdot x} a_{\lambda}(k)\right] d^{3} k, \\
& B(x)=-\frac{i}{2 \pi} \sum_{\lambda=1,2} \int|k|^{1 / 2}\left(\frac{k}{|k|} \wedge \epsilon_{\lambda}(k)\right) \rho(k)\left[e^{-i k \cdot x} a_{\lambda}^{*}(k)-e^{i k \cdot x} a_{\lambda}(k)\right] d^{3} k,
\end{aligned}
$$

où $\epsilon_{1}(k), \epsilon_{2}(k)$ sont des vecteurs de polarisation, $\epsilon_{\lambda}=\left(\epsilon_{\lambda}^{1}, \epsilon_{\lambda}^{2}, \epsilon_{\lambda}^{3}\right)$, satisfaisant $\epsilon_{\lambda}(k) \cdot \epsilon_{\lambda^{\prime}}(k)=\delta_{\lambda \lambda^{\prime}}$ et $k \cdot \epsilon_{\lambda}(k)=0$. La fonction $\rho$ est une fonction de troncature ultraviolette, choisie à valeurs réelles, et telle que

$$
\int_{|k| \leq 1} \frac{|\rho(k)|^{2}}{|k|^{2}} d^{3} k+\int_{|k| \geq 1}|k||\rho(k)|^{2} d^{3} k<\infty
$$


Enfin l'opérateur hamiltonien pour les photons en jauge de Coulomb est donné par

$$
H_{\mathrm{ph}}=\sum_{\lambda=1,2} \int|k| a_{\lambda}^{*}(k) a_{\lambda}(k) d^{3} k .
$$

Dans (1.2) et (1.4), $a_{\lambda}^{*}(k)$ et $a_{\lambda}(k)$ sont les opérateurs usuels de création et d'annihilation obéissant aux relations canoniques de commutation $\left(a^{\#}=a^{*}\right.$ ou $\left.a\right)$ :

$$
\left[a_{\lambda}^{\#}(k), a_{\lambda^{\prime}}^{\#}\left(k^{\prime}\right)\right]=0 \quad, \quad\left[a_{\lambda}(k), a_{\lambda^{\prime}}^{*}\left(k^{\prime}\right)\right]=\delta_{\lambda \lambda^{\prime}} \delta\left(k-k^{\prime}\right) .
$$

L'opérateur $H_{g}$ est invariant par translation dans la direction $x_{3}$, dans le sens où il commute formellement avec l'opérateur $P_{3}=p_{3}+\mathrm{d} \Gamma\left(k_{3}\right)$, où $\mathrm{d} \Gamma\left(k_{3}\right)$ est la seconde quantification de l'opérateur de multiplication par $k_{3} \in \mathbb{R}$. Aussi $H_{g}$ admet une décomposition en intégrale directe, $H_{g} \simeq$ $\int_{\mathbb{R}}^{\oplus} H_{g}\left(P_{3}\right) d P_{3}$, où $H_{g}\left(P_{3}\right)$ opère dans $\mathcal{H}=L^{2}\left(\mathbb{R}^{2} \times \mathbb{Z}_{2}\right) \otimes \mathcal{H}_{\mathrm{ph}}$, et (voir [3])

$$
\begin{aligned}
H_{g}\left(P_{3}\right)= & \frac{1}{2 m} \sum_{j=1,2}\left(p_{j}-e a_{j}\left(x^{\prime}\right)-g A_{j}\left(x^{\prime}, 0\right)\right)^{2}+\frac{1}{2 m}\left(P_{3}-\mathrm{d} \Gamma\left(k_{3}\right)-g A_{3}\left(x^{\prime}, 0\right)\right)^{2} \\
& -\frac{e}{2 m} \sigma_{3} b\left(x^{\prime}\right)-\frac{g}{2 m} \sigma \cdot B\left(x^{\prime}, 0\right)+V\left(x^{\prime}\right)+H_{\mathrm{ph} .}
\end{aligned}
$$

Soient $h(b, V)=\sum_{j=1,2} \frac{1}{2 m}\left(p_{j}-e a_{j}\left(x^{\prime}\right)\right)^{2}-\frac{e}{2 m} \sigma_{3} b\left(x^{\prime}\right)+V\left(x^{\prime}\right)$ et $e_{0}=\inf \sigma(h(b, V))$. Nous supposons que $b$ et $V$ sont choisis de telle façon que $e_{0}$ est une valeur propre isolée et de multiplicité finie (nous renvoyons à $[5,13,17,18]$ pour des choix possibles de couples $(b, V)$ satisfaisant cette propriété). Nous faisons de plus l'hypothèse suivante :

$\left(\mathbf{H}_{\mathbf{0}}\right) e_{0}$ est une valeur propre isolée de multiplicité 1.

Nous posons également $e_{1}=\inf \left[\sigma(h(b, V)) \backslash\left\{e_{0}\right\}\right]$. Définissons $H_{g, \sigma}\left(P_{3}\right)$ l'opérateur obtenu en introduisant dans $H_{g}\left(P_{3}\right)$ une troncature infrarouge, c'est-à-dire en remplaçant l'intégrale sur $\mathbb{R}^{3}$ définissant $A(x)$ dans (1.2) par l'intégrale sur $\left\{k \in \mathbb{R}^{3},|k| \geq \sigma\right\}$. Il est établi dans [3] que, pour $g$ et $P_{3}$ suffisamment petits, et pour tout $\sigma \geq 0, H_{g, \sigma}\left(P_{3}\right)$ est auto-adjoint et semi-borné inférieurement. Nous notons $E_{g, \sigma}\left(P_{3}\right)=\inf \sigma\left(H_{g, \sigma}\left(P_{3}\right)\right)$ l'infimum du spectre de $H_{g, \sigma}\left(P_{3}\right)$ pour $\sigma>0$, et $E_{g}\left(P_{3}\right)=$ $\inf \sigma\left(H_{g}\left(P_{3}\right)\right)$ pour $\sigma=0$. D'après [3], pour tout $\sigma>0, H_{g, \sigma}\left(P_{3}\right)$ possède un état fondamental $\Phi_{g, \sigma}\left(P_{3}\right)$, et, si l'on suppose de plus l'hypothèse $\left(\mathbf{H}_{\mathbf{0}}\right)$ vérifiée, $\Phi_{g, \sigma}\left(P_{3}\right)$ est non dégénéré.

\section{Résultats et remarques}

Notre résultat principal (voir le théorème 2.3 plus bas) fournit une condition nécessaire et suffisante de l'existence d'un état fondamental pour $H_{g}\left(P_{3}\right)$. L'une des propriétés cruciales que nous utilisons pour obtenir ce résultat est la régularité de l'application $P_{3} \mapsto E_{g}\left(P_{3}\right)$.

Proposition 2.1 Supposons l'hypothèse $\left(\mathbf{H}_{\mathbf{0}}\right)$ satisfaite. Alors il existe $g_{0}>0$ et $P_{0}>0$ tels que pour tout $0<|g| \leq g_{0}$, pour tous $P_{3}, k_{3} \in \mathbb{R}$ tels que $\left|P_{3}\right| \leq P_{0},\left|P_{3}+k_{3}\right| \leq P_{0}$, pour tout $0 \leq \sigma \leq\left(e_{1}-e_{0}\right) / 2$, pour tout $\delta>0$,

$$
\left|E_{g, \sigma}^{\prime}\left(P_{3}+k_{3}\right)-E_{g, \sigma}^{\prime}\left(P_{3}\right)\right| \leq \mathrm{C}_{\delta}\left|k_{3}\right|^{\frac{1}{4}-\delta},
$$

où $\mathrm{C}_{\delta}$ est une constante dépendant de $\delta$ mais ne dépendant pas de $\sigma$. 


\section{Remarques 2.2}

1. Le cas d'un électron libre interagissant avec le champ électromagnétique quantifié a été étudié récemment (cf [7, 8]). Le modèle correspondant présente des similarités avec le nôtre, dans la mesure où il est invariant par translation et conduit à l'étude d'un Hamiltonien $H_{g}(P)$ pour une impulsion totale fixée $P \in \mathbb{R}^{3}$. Il est toutefois à noter que pour ce modèle où un seul électron est considéré, $H_{g}(P)$ ne contient pas de partie électronique $h(b, V)\left(H_{g}(P)\right.$ agit dans $\mathcal{H}_{\mathrm{ph}}$ uniquement), ce qui, dans une certaine mesure, simplifie l'étude par rapport au modèle envisagé ici. Dans [7], pour un électron avec spin, le caractère $\mathrm{C}^{2}$ de l'application $P \mapsto E_{g}(P)=\inf \sigma\left(H_{g}(P)\right)$ est obtenu à partir d'une méthode basée sur l'utilisation d'un groupe de renormalisation (voir aussi [6]). L'auteur montre de plus que $\nabla E_{g}(P)=0$ si et seulement si $P=0$. Dans [8], à partir du travail antérieur de $A$. Pizzo sur le modèle de Nelson, [16], il est établi que $P \mapsto E_{g}(P)$ est de classe $\mathrm{C}^{1+\delta}$ pour tout $0 \leq \delta<1 / 4$. Nous avons pu adapter cette dernière méthode à notre modèle.

2. La preuve de la proposition 2.1 s'adapte au cas des atomes et des ions habillés non relativistes en interaction avec le champ électromagnétique quantifié (voir [1]), mais toujours sous une hypothèse de simplicité du type de $\left.\mathbf{( H}_{\mathbf{0}}\right)$. Le problème paraît plus difficile dans le cas dégénéré.

Posons $\Phi(h)=\left(a^{*}(h)+a(h)\right) / \sqrt{2}$ pour $h \in \mathrm{L}^{2}\left(\mathbb{R}^{3} \times \mathbb{Z}_{2}\right)$, où $a^{*}(h)=\sum_{\lambda=1,2} \int h(k, \lambda) a_{\lambda}^{*}(k) d^{3} k$ et $a(h)=\sum_{\lambda=1,2} \int \bar{h}(k, \lambda) a_{\lambda}(k) d^{3} k$. Le produit scalaire dans $\mathrm{L}^{2}\left(\mathbb{R}^{3} \times \mathbb{Z}_{2}\right)$ est défini par $\left(h_{1}, h_{2}\right)=$ $\sum_{\lambda=1,2} \int \bar{h}_{1}(k, \lambda) h_{2}(k, \lambda) d^{3} k$. Soit $W(h)=e^{i \Phi(h)}$ l'opérateur de Weyl, et soit $f: \mathbb{R}^{3} \times \mathbb{Z}_{2} \rightarrow \mathbb{C}^{\text {la }}$ fonction définie par

$$
f(k, \lambda)=\frac{g}{2 m} \frac{\rho(k) \epsilon_{\lambda}^{3}(k)}{k_{3}|k|^{1 / 2}} \frac{E_{g}\left(P_{3}-k_{3}\right)-E_{g}\left(P_{3}\right)}{E_{g}\left(P_{3}-k_{3}\right)-E_{g}\left(P_{3}\right)+|k|} .
$$

Notons que, si $P_{3} \mapsto E_{g}\left(P_{3}\right)$ est de classe $\mathrm{C}^{1+\alpha}$ avec $\alpha>0$, en utilisant le fait que pour $g$ et $P_{3}$ suffisamment petits, $E_{g}\left(P_{3}-k_{3}\right)-E_{g}\left(P_{3}\right) \geq-3|k| / 4$ (cf. $[3$, Lemma 4.3$\left.]\right)$, on a $f \in \mathrm{L}^{2}\left(\mathbb{R}^{3} \times \mathbb{Z}_{2}\right)$ si et seulement si $E_{g}^{\prime}\left(P_{3}\right)=0$. Introduisons, de la même façon que dans [4], un opérateur "renormalisé" $H_{g}^{\text {ren }}\left(P_{3}\right)$ à partir de l'expression formelle $H_{g}^{\text {ren }}\left(P_{3}\right)=W(i f) H_{g}\left(P_{3}\right) W(i f)^{*}$. Nous obtenons (voir par exemple [9]) :

$$
\begin{aligned}
H_{g}^{\mathrm{ren}}\left(P_{3}\right)= & \frac{1}{2 m} \sum_{j=1,2}\left(p_{j}-e a_{j}\left(x^{\prime}\right)-g A_{j}\left(x^{\prime}, 0\right)+g \operatorname{Re}\left(h_{j}\left(x^{\prime}\right), f\right)\right)^{2} \\
& +\frac{1}{2 m}\left(P_{3}-\mathrm{d} \Gamma\left(k_{3}\right)+\Phi\left(k_{3} f\right)+\frac{1}{2}\left(k_{3} f, f\right)-g A_{3}\left(x^{\prime}, 0\right)+g \operatorname{R} e\left(h_{3}\left(x^{\prime}\right), f\right)\right)^{2} \\
& -\frac{e}{2 m} \sigma_{3} b\left(x^{\prime}\right)-\frac{g}{2 m} \sigma \cdot B\left(x^{\prime}, 0\right)+V\left(x^{\prime}\right)+H_{\mathrm{ph}}-\Phi(|k| f)-\frac{1}{2}(|k| f, f),
\end{aligned}
$$

où l'on a posé $A_{j}\left(x^{\prime}, 0\right)=\Phi\left(h_{j}\left(x^{\prime}\right)\right)$. Remarquons que $H_{g}^{\text {ren }}\left(P_{3}\right)$ est unitairement équivalent à $H_{g}\left(P_{3}\right)$ si et seulement si $f \in \mathrm{L}^{2}\left(\mathbb{R}^{3} \times \mathbb{Z}_{2}\right)$. Notre principal résultat est alors :

Théorème 2.3 Supposons l'hypothèse $\left(\mathbf{H}_{\mathbf{0}}\right)$ satisfaite. Alors il existe $g_{0}>0$ et $P_{0}>0$ tels que pour tous $0<|g| \leq g_{0}$ et $0<\left|P_{3}\right| \leq P_{0}$,

(i) $H_{g}\left(P_{3}\right)$ possède un état fondamental si et seulement si $E_{g}^{\prime}\left(P_{3}\right)=0$.

(ii) $H_{g}^{\mathrm{ren}}\left(P_{3}\right)$ possède un état fondamental. 


\section{Remarques 2.4}

1. L'inégalité (2.1) est utilisée à la fois dans la preuve de (i) et dans celle de (ii). Plus précisément, nous utilisons (2.1) avec $\sigma=0$ afin d'obtenir l'absence d'état fondamental de $H_{g}\left(P_{3}\right)$ lorsque $E_{g}^{\prime}\left(P_{3}\right) \neq 0$ : nous basant sur [10, Lemme 2.6], nous obtenons une contradiction en supposant l'existence d'un état fondamental $\Phi_{g}\left(P_{3}\right)$, grâce à la propriété

$$
(k, \lambda) \mapsto\left\|\left[a_{\lambda}(k)-f(k, \lambda)\right] \Phi_{g}\left(P_{3}\right)\right\| \in \mathrm{L}^{2}\left(\mathbb{R}^{3} \times \mathbb{Z}_{2}\right) .
$$

L'existence d'un état fondamental pour $H_{g}^{\mathrm{ren}}\left(P_{3}\right)$, ou pour $H_{g}\left(P_{3}\right)$ lorsque $E^{\prime}\left(P_{3}\right)=0$, s'obtient quant à elle de la même façon que dans [3], en utilisant de plus (2.1) avec $\sigma>0$ pour contrôler le nombre de photons dans l'état fondamental de $H_{g, \sigma}\left(P_{3}\right)$.

2. La preuve du théorème 2.3 s'adapte au cas des atomes et des ions (voir [1]), en remplaçant la condition $E_{g}^{\prime}\left(P_{3}\right)=0$ dans $(i)$ par $Q \nabla E_{g}(P)=0$, où $Q$ représente la charge totale du système atomique. Le cas des atomes, $Q=0$, est traité dans [2]. Pour toute valeur de la constante de couplage, l'existence d'un état fondamental pour $Q=0$ est également obtenue dans [14], en adaptant la méthode de [11], mais sous l'hypothèse $E_{g}(P) \geq E_{g}(0)$ qui, jusqu'à maintenant, n'a pas pu être vérifiée pour une valeur quelconque de $g$. Dans [12], les auteurs montrent l'absence d'état fondamental pour $H_{g}(P)$ dans le cas $Q<0$, en supposant que $\nabla E_{g}(P)$ est différent de 0 . Ainsi, par rapport à ces résultats, la méthode que nous employons permet en plus d'obtenir l'existence d'un état fondamental pour $H_{g}^{\text {ren }}(P)$ et pour $H_{g}(P)$ lorsque $\nabla E_{g}(P)=0$.

3. Si $E_{g}^{\prime}\left(P_{3}\right) \neq 0$, (ii) fournit l'existence d'un état fondamental dans une représentation non équivalente à la représentation Fock des relations canoniques de commutation, comme dans le cas du modèle de Nelson (pour un système atomique non invariant par translation, voir [4], [10], [15]).

\section{References}

[1] L. Amour, J. Faupin, B. Grébert, and J.-C. Guillot. The infrared problem for the dressed mobile ions. In preparation.

[2] L. Amour, B. Grébert, and J.-C. Guillot. The dressed mobile atoms and ions. J. Math. Pures Appl. (9), 86(3):177-200, 2006.

[3] L. Amour, B. Grébert, and J.-C. Guillot. The dressed nonrelativistic electron in a magnetic field. Math. Methods Appl. Sci., 29(10):1121-1146, 2006.

[4] A. Arai. Ground state of the massless Nelson model without infrared cutoff in a non-Fock representation. Rev. Math. Phys., 13(9):1075-1094, 2001.

[5] J. Avron, I. Herbst, and B. Simon. Schrödinger operators with magnetic fields. I. General interactions. Duke Math. J., 45(4):847-883, 1978.

[6] V. Bach, T. Chen, J. Fröhlich, and I. M. Sigal. The renormalized electron mass in non-relativistic quantum electrodynamics. J. Funct. Anal., 243(2):426-535, 2007.

[7] T. Chen. Infrared renormalization in non-relativistic qed and scaling criticality. J. Funct. Anal., 2008. doi:10.1016/j.jfa.2008.01.001.

[8] T. Chen, J. Fröhlich, and A. Pizzo. Infraparticle Scattering States in Non-Relativistic QED II. Mass Shell Properties. arxiv.org, math-ph/07092812, 2007.

[9] J. Dereziński and C. Gérard. Asymptotic completeness in quantum field theory. Massive Pauli-Fierz Hamiltonians. Rev. Math. Phys., 11(4):383-450, 1999.

[10] J. Dereziński and C. Gérard. Scattering theory of infrared divergent Pauli-Fierz Hamiltonians. Ann. Henri Poincaré, 5(3):523-577, 2004. 
[11] M. Griesemer, E. H. Lieb, and M. Loss. Ground states in non-relativistic quantum electrodynamics. Invent. Math., 145(3):557-595, 2001.

[12] D. Hasler and I. Herbst. Absence of Ground States for a Class of Translation Invariant Models of Non-relativistic QED. arxiv.org, math-ph/0702096, 2007.

[13] A. Iwatsuka and H. Tamura. Asymptotics distribution of eigenvalues for Pauli operators with non constant magnetic fields. Duke Math. J., 93:535-574, 1998.

[14] M. Loss, T. Miyao, and H. Spohn. Lowest energy states in nonrelativistic QED: atoms and ions in motion. $J$. Funct. Anal., 243(2):353-393, 2007.

[15] A. Panati. Existence and non existence of a ground state for the massless Nelson model under binding condition. arxiv.org, math-ph/0609065, 2006.

[16] A. Pizzo. One-particle (improper) states in Nelson's massless model. Ann. Henri Poincaré, 4(3):439-486, 2003.

[17] G.D. Raikov. Eigenvalue asymptotics for the Pauli operator in strong non-constant magnetic fields. Ann. Inst. Fourier, 49:1603-1636, 1999.

[18] A. V. Sobolev. On the Lieb-Thirring estimates for the Pauli operator. Duke Math. J., 82(3):607-635, 1996. 